\title{
ASPECTOS FUNDAMENTALES DE LA RESPONSABILIDAD CIVIL ALEMANA EN EL ÁMBITO DE LA CIRUGÍA ESTÉTICA
}

\author{
FUNDAMENTALS OF GERMAN \\ MEDICAL LIABILITY LAW \\ IN THE FIELD OF AESTHETIC SURGERY
}

\author{
Dr. iuris Markus Finn
}

\section{RESUMEN}

El Derecho alemán de responsabilidad médica hace una distinción entre la responsabilidad por errores de tratamiento y la responsabilidad por falta de información. En las intervenciones de la cirugía estética se trata, por regla general, de un error de tratamiento cuando el médico tratante quede por debajo del estándar vigente en el respectivo ramo especial de la cirugía estética plástica. En cambio, en el caso de las faltas de información la responsabilidad (delictual) se basa en la lesión corporal que, según la jurisprudencia de los tribunales alemanes, es inherente a toda intervención médica en el estado corporal o de salud del paciente. No obstante, la lesión corporal no se estima antijurídica si este, después de haber sido debidamente informado, ha dado su consentimiento válido (informed consent). El consentimiento válido, del cual la parte tratante deberá dar pruebas en el juicio, puede justificar la lesión corporal. En las operaciones estéticas es de importancia práctica, ante todo, la responsabilidad por insuficiencia de información sobre los riesgos de la respectiva intervención. Si una operación estética carece de indicación médica-como ocurre con frecuencia- los requisitos relativos a la información del paciente son especialmente rigurosos. En este sentido, la jurisprudencia exige una información extensa, intensa y a veces "desconsiderada" sobre los riesgos inminentes, tratándose de compensar en cierto modo la "indicación meramente estética” por medio del consentimiento cualificado del paciente

* Profesor titular de la cátedra de Derecho Sanitario de la Hochschule Hof, Profesor encargado de la Charité - Universitätsmedizin Berlín. Dirección postal: Hochschule Hof, Alfons-Goppel-Platz 1, D-95028 Hof, Alemania. Correo electrónico: markus.finn@hofuniversity.de. Artículo recibido el 17 de abril de 2015 y aceptado para su publicación el 28 de mayo de 2015. 
después de su información extensa (intensely informed consent). En caso de comprobarse que la información ha sido insuficiente, los médicos y hospitales pueden resultar responsables de los daños y perjuicios y obligados a pagar indemnización por daños personales.

Palabras claves: responsabilidad médica, cirugía estética, falta de información, error de tratamiento, contrato de tratamiento, promesa de resultado, informed consent, causalidad, indemnización de daños y perjuicios, indemnización por daños personales, operación de cirugía estética, información sobre riesgos.

Abstract

The German medical liability law makes a distinction between the responsibility for treatment mistakes and responsibility for lack of information. Interventions in aesthetic surgery is, as a rule, a medical practice when the physician is below the standard in force in the respective special field of aesthetic plastic surgery. However, in the case of lack of information liability (tort) is based on bodily injury, according to the jurisdiction of German courts is inherent in any medical intervention in body or health status of the patient. However, bodily injury is not considered unlawful if the patient, after having been duly informed, has given valid consent (informed consent). Informed consent, which the treating party should give evidence at trial, can justify the bodily injury. In the aesthetic operations it is of practical importance, above all, the responsibility for insufficient information on the risks of the respective intervention. If aesthetic surgery, as no medical indication occurs often, the requirements for patient information are particularly stringent. In this sense, the jurisdiction requires a extensive, intense and sometimes "thoughtless" information about imminent risks, in the case of offset to some extent the purely "aesthetic indication" by the qualified patient consent after extensive information (intensely informed consent ). If it is found that the information was insufficient, doctors and hospitals may be liable for damages and ordered to pay compensation for personal injury.

Keywords: medical liability, cosmetic surgery, lack of information, error treatment, treatment contract, promising result, informed consent, causation, compensation for damages, compensation for personal injury, plastic surgery, risk information. 


\section{INTRODUCCIÓN}

A partir del llamado escándalo de la silicona, muchas personas en Europa han llegado a tomar real conciencia de la dimensión de la responsabilidad civil que tiene la cirugía estética. A principios de 2010 se divulgó que los implantes mamarios del fabricante francés PIP no estaban rellenos del gel de silicona previsto especialmente para uso médico, sino de una silicona industrial de calidad inferior. Aparte del probablemente elevado riesgo de agrietarse, es de temer que la silicona industrial se difunda en el cuerpo. Incluso sin formación de grietas, puede ocurrir el llamado Gel-Bleeding. La silicona salida puede tanto provocar reacciones en el tejido como distribuirse en el cuerpo y acumularse, por ejemplo, en los ganglios linfáticos axilares.

Cuando en marzo de 2010 la autoridad francesa de control ${ }^{1}$ descubrió que el fabricante PIP empleaba silicona industrial, se prohibieron en toda Europa la distribución y utilización de sus implantes mamarios. En lo sucesivo, la autoridad federal suprema competente para productos sanitarios en Alemania ${ }^{2}$ emitió recomendaciones destinadas a evitar peligros para la salud $^{3}$. Últimamente se recomendó, como medida precautoria, la explantación de los implantes en cuestión, en que la urgencia de tal medida dependía de cuánto tiempo la paciente llevaba ya implantado el producto. Muchas mujeres se hicieron retirar sus implantes mamarios defectuosos ${ }^{4}$.

El escándalo pudo producirse porque el sistema de examen y vigilancia de los sistemas de calidad fracasó en el caso de la empresa PIP a causa de actos fraudulentos ${ }^{5}$ del fundador de la empresa y varios colaboradores. Antes de las inspecciones anunciadas de las entidades de vigilancia, se procuró siempre hacer desaparecer a tiempo todo material sospechoso y la correspondiente documentación. Después de revelarse el escándalo, la empresa fabricante declaró su concurso, siendo uno de los motivos, y no el menos importante, por el que tanto en Alemania ${ }^{6}$ como en Francia ${ }^{7}$ las

${ }^{1}$ Afssaps, ahora ANSM.

${ }^{2}$ El BfArM.

${ }^{3}$ Para más detalles al respecto, véase las recomendaciones del BfArM, disponibles en www.bfarm.de/SharedDocs/Risikoinformationen/Medizinprodukte/DE/Silikon_ Brustimplantate_PIP.html. [Fecha de consulta 7 de abril de 2015].

${ }^{4}$ Las mujeres afectadas -en caso de tener seguro obligatorio de enfermedad- deberán participar en los gastos por ello originados con un importe "adecuado" (§ 52 párrafo 2 SGB V), en detalle, véase SG Berlin (2013).

${ }^{5}$ Sancionados ante tribunales penales franceses.

${ }^{6}$ Véase OLG Zweibrücken (2014). Desestimación de la demanda, recientemente BGH (2015): resolución de remisión al Tribunal de Justicia de la Unión Europea.

${ }^{7}$ Véase Tribunal de Commerce Toulon (2013): condena al pago de $3.000 €$ por cada víctima en concepto de indemnización de daños y perjuicios. Anulado por la Cour d'Appel de Aix en Provence (2015), véase Fröding (2013), p. 162 y ss. 
pacientes agraviadas dirigieron sus reclamaciones judiciales por daños y perjuicios también contra las empresas de vigilancia que habían realizado en PIP exclusivamente inspecciones anunciadas, si bien hasta la fecha no hay sentencia en firme.

Por último, hay ya demandas entabladas por pacientes afectadas contra los médicos y clínicas tratantes, sin que en Alemania -según se ha podido apreciar- se haya publicado aún ninguna resolución judicial sobre este tema. Solo han sido desestimadas, según un comunicado de prensa del Tribunal Regional de Karlsruhe ${ }^{8}$, dos demandas de indemnización de daños y perjuicios entabladas contra una clínica por estimar que los médicos podían confiar en la certificación del fabricante y que, en todo caso, en 2007, cuando fueron colocados los implantes de las demandantes del caso, todavía no existían indicios para poner en duda la calidad de los implantes de PIP.

No obstante, los médicos y clínicas no siempre quedan resguardados de los juicios de responsabilidad en el contexto de la cirugía estética. Mientras que en el escándalo de la silicona, al parecer, no se le ha podido imputar a la parte tratante ni siquiera la falta de desconfianza, los juicios por intervenciones en este ámbito tratan frecuentemente de serios errores de tratamiento o faltas de información. Para discutir los problemas específicos de las intervenciones estéticas sin indicación médica -es decir, de las intervenciones meramente cosméticas- (véase más adelante III), resulta oportuno resumir, en primer lugar, los aspectos fundamentales del derecho de responsabilidad médica en Alemania (véase a continuación II).

\section{Aspectos Fundamentales \\ DEL DERECHO DE RESPONSABILIDAD MÉDiCa en Alemania ${ }^{9}$ \\ 1. Desarrollo del derecho de responsabilidad médica por la jurisprudencia}

Durante varias décadas, el Derecho de Responsabilidad Médica en Alemania ha sido desarrollado y determinado en lo esencial por la Sala de lo Civil VI del BGH -el tribunal supremo alemán en materia civil-. En este

${ }^{8}$ Comunicado de prensa con fecha del 7 de junio de 2013, disponibles en Internet en www.landgericht-karlsruhe.de/pb/,Lde/1160639/?LISTPAGE=1160343 [fecha de consulta 7 de abril de 2015].

${ }^{9}$ Este trabajo trata del Derecho alemán, véase en cuanto al Derecho chileno BARCiA (2010), Cárdenas (2010), De La Maza (2010), Domínguez (2010), Figueroa (2010), Pizarro (2013), Pizarro (2011), Pizarro (2010), Pizarro (2008), en relación con el Derecho español Pérez (2010) y Wiegner (2010). 
aspecto, la falta de regulaciones especiales no constituía ningún déficit, ya que las normas generales del Derecho de los Contratos y de los Delitos Civiles resultaron ser válidas y flexibles y permitieron a la jurisprudencia continuar estableciendo el Derecho de Responsabilidad Médica según los desarrollos de la Medicina y las opiniones de la sociedad ${ }^{10}$. Tratando de lograr un equilibrio adecuado entre los intereses de los pacientes, por un lado, y los de la parte tratante, por el otro, se creó un derecho de responsabilidad médica muy diferenciado que en parte, como compleja materia especial, ha quedado y sigue quedando reservado exclusivamente a profesionales de Derecho especializados. En el transcurso de los años se ha podido observar también un creciente nivel de las obligaciones de los médicos, tanto con respecto al tratamiento médico como a la información del paciente. Precisamente en el ámbito de la información de los pacientes, los requisitos establecidos han sido criticados como parcialmente exagerados, sobre todo teniendo en consideración la labor cotidiana del médico ${ }^{11}$.

La responsabilidad se basa tanto en el Derecho de los Contratos como en el de los Delitos Civiles. Sin embargo, ambas áreas han sido armonizadas en cuanto a los requisitos esenciales, tales como nivel de diligencia, requisitos de causalidad y daños reparables ${ }^{12}$. Ya apenas existen diferencias esenciales ${ }^{13}$.

\section{Codificación del contrato de tratamiento $y$ de las reglas de prueba en el $B G B$}

Con efectos a partir del 26 de febrero de 2013, por medio de la llamada Ley de Derechos del Paciente ${ }^{14}$ ha quedado codificado en el $B G B$ el contrato de tratamiento, incluyendo algunas obligaciones esenciales de médicos y demás personal tratante ${ }^{15}$ (véase $\$ \S 630 \mathrm{a}$ a $630 \mathrm{~g}$ del $B G B$ ). Asimismo, el $B G B$ incluye ahora, también, regulaciones relativas a la distribución de la carga probatoria para graves errores de tratamiento, entre otros (§ $630 \mathrm{~h}$ del $B G B$ ). Empero, no todas las diferenciaciones de la jurisprudencia han quedado reflejadas en el Derecho ahora codificado, orientándose la codificación parcial realizada del contrato de tratamiento y de las reglas

\footnotetext{
${ }^{10}$ Véase Katzenmeier (2014), p. 7.

${ }^{11}$ En detalle, véase Laufs, Katzenmeier y Lipp (2009), capítulo v, cons. $63^{\circ}$ y ss (con más pruebas).

${ }^{12}$ Véase Geiß/Greiner (2014), Einleitung, cons. $2^{\circ}$.

${ }^{13}$ Con respecto a una diferencia que aún permanece, pero menos importante véase Geiß/Greiner (2014), Einleitung, cons. $2^{\circ}$.

${ }^{14}$ Véase Gesetz zur Verbesserung der Rechte von Patientinnen und Patienten de 20 de febrero de 2013.

${ }^{15}$ Por ejemplo, odontólogos, psicoterapeutas psicológicos, comadronas, fisioterapeutas.
} 
de prueba, fundamentalmente, por el afán del legislador por establecer en forma de ley los principios ya antes reconocidos ${ }^{16}$. Por lo tanto, novedades substanciales fueron más bien una excepción. Asimismo, persistió en lo esencial inafectada por las nuevas normas jurídicas creadas la responsabilidad extracontractual por delito ${ }^{17}$.

\section{Principios de responsabilidad}

\section{a) Responsabilidad de doble vía: falta de información y error de tratamiento}

El Derecho alemán de responsabilidad médica tiene carácter de doble vía. Según muestra la experiencia, aparte de reprochar un error de tratamiento, en la mayoría de los juicios de responsabilidad médica se entabla también una reclamación por falta de información. Es decir, se le reprocha al médico no solo no haber tratado de forma debida sino que se le imputa también que, en todo caso, no ha informado debidamente. Uno de los motivos de los abogados de pacientes para este modo de proceder en el juicio civil es que en este, por principio, es el paciente a quien incumbe la carga de alegación y prueba del error de tratamiento, así como de la causalidad del error mismo por el daño reclamado. Pero, en muchos casos, a los agravia60 dos les es difícil probar especialmente la causalidad, ya que con frecuencia entran en consideración varias causas alternativas del daño, como pueden ser la enfermedad tratada, en casos de pacientes multimórbidos las otras enfermedades, sin olvidar un posible desarrollo fatal. Por eso, puede servir como "áncora de salvación" de la acción indemnizatoria la recriminación de que, de haber sido informado debidamente, el paciente no hubiera dado su consentimiento necesario para el tratamiento. En este caso, este tiene la ventaja de que la carga de alegación y prueba de la información y del consentimiento incumbe al médico.

b) Consentimiento válido del paciente informado (informed consent)

Independientemente de si existe o no un error de tratamiento, los médicos pueden resultar responsables de daños y perjuicios de pacientes por insuficiencia o falta de información a efectos de la autonomía del mismo. En caso de faltas de información, la responsabilidad (delictual) se basa en la lesión corporal ${ }^{18}$ que, según la jurisprudencia de los tribunales alemanes,

${ }^{16}$ Véase Boletín oficial del Bundestag (2012), 17/10488, pp. 1, 9 y passim.

${ }^{17}$ Véase, sobre todo, $\S \S 823,831,839$ del $B G B$.

${ }^{18}$ En caso de responsabilidad contractual, la infracción de deberes en el sentido del $\S 280$ párrafo 1 frase 1 del $B G B$. 
es inherente a toda intervención médica en el estado corporal o de salud del paciente ${ }^{19}$. Sin embargo, no se estima antijurídica la lesión corporal cuando este, después de haber sido debidamente informado, ha dado su consentimiento válido (informed consent). En el juicio, el consentimiento válido debe ser probado por la parte tratante como hecho justificativo ${ }^{20}$. Si por lo contrario, la información resulta ser insuficiente o no puede ser probada por el médico - por ejemplo, con documentos o, en su caso, testigos- falta el consentimiento válido. Entonces, la lesión corporal no es justificada y el médico puede resultar responsable de los daños y perjuicios y obligado a pagar indemnización por daños personales, que ocurren por la intervención médica. Esa responsibilidad por falta de información no depende de un error de tratamiento.

Los requisitos relativos al alcance de la información aumentan con los riesgos relacionados con la intervención ${ }^{21}$. En primer lugar, es decisiva la gravedad de los daños que la intervención puede provocar en la manera en que el paciente continuará su vida, así como la circunstancia de que un riesgo esté entrañado específicamente a la intervención, y solo en segundo lugar lo es la frecuencia de las consecuencias que, según la experiencia, conlleva el daño ${ }^{22}$. Hay que informar, también, sobre los riesgos poco frecuentes si en caso de llegar a producirse, la manera de continuar la vida del paciente resultaría gravemente afectada y si, a pesar de su poca frecuencia, son específicos de la intervención e inesperados por personas no expertas ${ }^{23}$. Puede ser necesario informar incluso sobre riesgos que ocurren "extremadamente raras veces" ${ }^{44}$ o cuando se trata de un coeficiente de riesgo a nivel de milésimas ${ }^{25}$. Huelga decir que la obligación de información se refiere solo a los riesgos conocidos en el momento del tratamiento. Los riesgos generales de operación, tales como embolias, trombosis, infecciones de heridas, etc. normalmente pueden darse por $\operatorname{conocidos}^{26}$.

${ }^{19}$ En cuanto al Derecho Penal, ya el Tribunal Supremo del Reich (RG) en el año 1894, RG (1894), y al Derecho Civil RG (1908) y BGH (1958).

${ }^{20}$ En cuanto a la responsabilidad contractual, véase también $\S 630$ h párrafo 2 frase 1 del $B G B$.

${ }^{21}$ Véase Laufs, Katzenmeier y Lipp (2009), Capítulo v, cons. $22^{\circ}$.

${ }^{22}$ Op. cit. y Geiß/Greiner (2014), capítulo C, cons. $22^{\circ}$.

${ }^{23}$ Véase, por ejemplo, BGH (2006b), p. 2109.

${ }^{24}$ Véase, por ejemplo, BGH (1979), p. 634, más pruebas en LaUfs, Katzenmeier y Lipp (2009), capítulo v, cons. $23^{\circ}$, n. 74.

${ }^{25} \mathrm{BGH}$ (1995), p. 781.

${ }^{26}$ Más detalles al respecto con pruebas de la jurisprudencia Geiß/Greiner (2014), capítulo C, cons. $47^{\circ}$. 
c) Estándar reconocido de la medicina especializada e indicación médica

Aparte de la responsabilidad por faltas de información, la responsabilidad por errores de tratamiento se basa en el incumplimiento del estándar reconocido de la medicina especializada a observar por principio por todo médico. El concepto del estándar reconocido de la medicina especializada comprende tres elementos relevantes: conocimiento científico, experiencia práctica y aceptación profesional ${ }^{27}$. En los juicios de responsabilidad, el tribunal normalmente no puede aclarar esta cuestión, sino ordenando la emisión de un dictamen pericial médico. De comprobarse por el tribunal la existencia de un error de tratamiento, por lo general consta también la culpa necesaria de la parte tratante (imprudencia o intencionalidad) ${ }^{28}$. Aunque en el Derecho alemán la responsabilidad está concebida como responsabilidad por culpa, se aplica un concepto objetivado de imprudencia, según el cual los médicos deben adoptar las medidas que desde el punto de vista profesional de su especialidad son de esperar de un profesional concienzudo y atento. Aun cuando la conducta sea subjetivamente excusable, no lo exculpa en el Derecho Civil.

La cuestión de si la intervención médica requiere una indicación médica para no ser calificada como error de tratamiento es controvertida ${ }^{29}$. 62 En la mayoría de los casos, de la falta de indicación médica no se deduce necesariamente la existencia de un error de tratamiento, a pesar de que a falta de tal indicación por regla general concurrirá un error de tratamiento. No obstante, en cuanto a la "indicación estética" de las operaciones de cirugía estética véase más adelante III. 2.

\section{d) Causalidad e inversión de la carga de la prueba}

Según los principios procesales generales, incumbe al agraviado la carga de alegación y prueba de la causalidad del error para el daño ocurrido. En caso de errores de tratamiento, por principio procede invertir la carga de la prueba en perjuicio del médico cuando este ha cometido un llamado "grave error de tratamiento" 30 . Según la doctrina sentada por el BGH, este es el caso cuando existe una infracción clara y fundamental de las reglas probadas del tratamiento o los conocimientos médicos consolidados que, según las circunstancias del caso concreto, desde el punto de vista objetivo ya no se considera comprensible y no es aceptable en absoluto que

${ }^{27}$ Katzenmeier (2002), p. 279.

${ }^{28} \S \S 276$ párrafo 1 frase 1 y párrafo 2, 280 párrafo 1 frase 2, 823 párrafo 1 del $B G B$.

${ }^{29}$ Más detalles sobre esta controversia en SpickноғF (2014); $§ 823$ del $B G B$, cons. $7^{\circ}$.

${ }^{30}$ Véase $§ 630$ h párrafo 5 frase $1 \mathrm{del} B G B$. 
sea cometida por un médico ${ }^{31}$. Si de manera general el error es indicado para provocar el daño ocurrido, al menos como una entre varias causas, el médico tiene que exculparse, es decir, debe rendir la contraprueba y acreditar que el daño de salud no ha sido provocado por el grave error de tratamiento ${ }^{32}$. Sin embargo, la experiencia muestra que en la práctica esta contraprueba está sujeta a dificultades apenas superables.

\section{PARTICULARIDADES DE LA RESPONSABILIDAD EN EL ÁMBITO DE LA CIRUGÍA ESTÉTICA}

\section{1. ¿Promesa de resultado del médico?}

En cuanto a los tratamientos curativos queda entendido que del contrato entre médico y paciente no se deriva la obligación de obtener un resultado en el sentido del contrato de obra ${ }^{33}$. En cambio, tratándose de una intervención estética no indicada desde el punto de vista médico, que a falta de dicha indicación médica no puede considerarse una intervención curativa $^{34}$, su tipología contractual es objetivo de vivas discusiones. La jurisprudencia ${ }^{35}$ y una parte de la literatura ${ }^{36}$ opinan que, por regla general, la parte tratante no responde del resultado de su prestación ni siquiera cuando se trata de una operación meramente cosmética, pues debido a circunstancias corporales imponderables el médico, obrando con máxima diligencia, no puede originar con seguridad un resultado y por eso no quiere prometer ningún resultado del tratamiento ${ }^{37}$. Por lo tanto, si es que

${ }^{31}$ Reiterada jurisprudencia, véase, por ejemplo, BGH (2011), p. 228.

${ }^{32}$ Véase BGH (2012), p. 2653.

${ }^{33}$ Véase ahora también $\S 630 \mathrm{~b}$ del $B G B$, que remite a las normas del contrato de servicios y no a las del contrato de obra.

${ }^{34}$ Véase Laufs y KERN (2010), § 38, cons. $27^{\circ}$.

${ }^{35}$ Véase, por ejemplo, OLG Sachsen-Anhalt (2012), cons. $7^{\circ}$ : incluso si se intenta conseguir un "resultado óptico perfecto".

${ }^{36}$ Véase Damm (2010), p. 647; Laufs y Kern (2010), § 38, cons. 27º Lorz (2007), pp. 78 y 252; Spickhoff en Spickhoff (2014), § 630a del BGB, cons. $8^{\circ}$.

${ }^{37}$ En el Derecho español, al contrario, existe una "obligación de resultado" en el ámbito de medicina satisfactoria -cirugía estética, entre otros-, así WIEGNER (2010), p. 65, también PÉREz (2010), pp. 151-152 (“obligación de resultado, que se aproxima al contrato de obra”) con pruebas de la doctrina y de la jurisprudencia del Tribunal Supremo, también pp. 162-163 (n. $43^{\circ}$ ). Véase con relación al Derecho chileno Pizarro (2013), p. 625 y ss., que diferencia distintos casos -calificación de ciertos obligaciones como resultado; con relación a los criterios relevantes para diferenciar véase p. 634 y ss.- con pruebas de la doctrina y de la jurisprudencia, referente a la cirugía estética véase p. 632 y ss., también Domínguez (2010) y CÁrdenas (2010). 
puede haber un contrato de obra, serán casos excepcionales ${ }^{38}$, sobre todo cuando haya una promesa de resultado del médico, estando su existencia sujeta a rigurosas condiciones. Por ejemplo, el Tribunal Regional Superior de Hamburgo ${ }^{39}$ no calificó como promesa de resultado la afirmación de un médico de que después de la abdominoplastia planificada la paciente iba a "lucir fantástica". Hay otras opiniones en la literatura ${ }^{40}$ que defienden la clasificación como contrato de obra, pero estos autores conceden al médico la posibilidad de derogar la responsabilidad agravada ${ }^{41}$. De este modo, entre esta posición y la opinión contraria se reduce a la inversión de la relación entre regla y excepción.

Por consiguiente, según la opinión predominante el médico tiene el deber de esforzarse por conseguir el resultado intentado. En cuanto al contenido, el acuerdo contractual se refiere a un margen de fluctuación dentro del cual se ubicará la apariencia corporal modificada ${ }^{42}$.

\section{2. "Indicación estética" en vez de indicación médica}

A diferencia de la intervención curativa, las operaciones cosméticas con el único objetivo de "embellecer" carecen de una indicación médica. A veces se dice que las operaciones de cirugía estética tienen una "indicación sui generis" o "indicación estética". No obstante, esta no infiere la justificación especial de un objetivo curativo ${ }^{43}$, ya que una "indicación meramente estética" no se ajusta a la finalidad de salud, sino a la orientación por la "belleza" 4 .

Esto nos lleva a la cuestión de principio, de si los déficits de la indicación médica pueden compensarse por medio de aumentos de información y, de ser así, en qué medida ${ }^{45}$. El consentimiento de pacientes dado después de haber sido informados de forma especialmente intensa debe adquirir una mayor importancia y poner en primer plano el precepto jurídico de volenti non fit iniuria. Analizándolo con detenimiento, la cirugía estética cae en el dilema entre la autonomía del paciente y el paternalismo: ¿Se puede o, en su caso, se debe proteger a los individuos contra ellos mis-

${ }^{38}$ Véase LAufs y Kern (2010), § 38, cons. $28^{\circ}$.

${ }^{39}$ Véase OLG Hamburg (2005), p. 873.

${ }^{40}$ Pruebas de Damm (2010), p. 647 , n. $55^{\circ}$ y $56^{\circ}$.

${ }^{41}$ Véase Eberbach (2008), p. 335.

${ }^{42}$ Véase LoRz (2007), p. 72.

${ }^{43}$ Según muestra la experiencia, en espera de un resultado curativo los pacientes están dispuestos a correr ciertos riesgos.

${ }^{44}$ Véase Damm (2010), pp. 644 y 652, opinión contraria LoRz (2007), p. 175, que, aparte de la salud, considera la belleza una finalidad por principio legítima del tratamiento.

${ }^{45}$ Véase Damm (2010), p. 653. 
mos estableciendo exigencias jurídicas agravadas? Aparte del aspecto de la regulación estatal, en Alemania tampoco podemos olvidarnos de los intereses privados, probablemente muy poderosos y con potenciales efectos limitadores de la autonomía de terceros ${ }^{46}$. Por esto, cuando y en la medida en que la "industria de la belleza" sea capaz de limitar la autonomía del paciente, en determinados casos puede resultar aconsejable una regulación estatal.

Con vistas a la relación entre indicación e información, es aceptada una fórmula recíproca, es decir, que exista una proporción inversa entre la indicación para la intervención, por un lado, y la intensidad de la información obligada, por el otro ${ }^{47}$. Por tanto, aplica el principio de que el paciente debe ser informado sobre las perspectivas de éxito y eventuales consecuencias nocivas de una intervención médica de forma tanto más extensa e insistente cuanto menos necesaria es esta desde el punto de vista médico, o sea, que no sirve, o no sirve, en primer lugar, para curar una dolencia corporal, sino, más bien, para satisfacer una necesidad psíquica y estética ${ }^{48}$.

Dentro de las intervenciones estéticas comprendidas en un sentido amplio puede resultar conveniente hacer graduaciones, ya que en el ámbito de la cirugía estética ellas no siempre son de carácter meramente cosmético sin ninguna indicación médica. A veces es imposible delimitar con exactitud las intervenciones con indicación médica de las que no la tienen, debiéndose constatar que los límites son difusos: desde la eliminación de una limitación funcional física ${ }^{49}$, pasando por la supresión quirúrgica de una deformación desfigurante e intervenciones quirúrgicas por alteraciones psíquicas ${ }^{50}$ hasta el mero embellecimiento de formas corporales que sin intervención alguna son normales ${ }^{51}$. En este contexto se confrontan las categorías de la medicina indicada y de la medicina del deseo, entre las cuales se sitúan otras categorías más de procedimientos, tales como la llamada cesárea deseada cada vez más aplicada para determinar exactamente la fecha del parto por medio de la operación cesárea, debiéndose graduar la intensidad de la información según la fórmula recíproca.

${ }^{46}$ Para más detalles véase DAMm (2010), p. 654 con más pruebas.

${ }^{47}$ Véase op. cit., pp. 650-652.

${ }^{48}$ Así expresamente BGH (2006b), p. 2108, véase también Katzenmeier (2002), p. 328.

${ }^{49}$ Por ejemplo, operación del tabique nasal para tratar una alteración crónica de la respiración nasal.

${ }^{50}$ Por ejemplo, corrección de orejas en caso de otapostasis.

${ }^{51}$ Véase LoRz (2007), p. 38 y ss., allí en relación con el derecho del seguro de enfermedad. 


\section{Consentimiento válido del paciente extensamente informado $0^{52}$ (intensely informed consent)}

En el ámbito de la cirugía estética, los tribunales alemanes establecen requisitos especialmente rigurosos con respecto a la información del paciente $^{53}$. El motivo de estos requisitos relativos a la información del paciente radica en que la falta de indicación médica ${ }^{54}$ es tratada de compensar $^{55}$ en la medida de lo posible ${ }^{56}$.

Los requisitos "muy rigurosos" establecidos por el BGH pueden ser concretizados del modo siguiente: Se le debe dar a conocer al paciente cuáles mejoramientos puede esperar en el caso más favorable, advirtiéndolo, a la vez, claramente de los eventuales riesgos con el fin de que pueda ponderar con esmero si quiere asumir la posibilidad de un fracaso de la intervención, en todo caso lesiva para él e, incluso, posibles perjuicios de salud, aunque estos no signifiquen más que una consecuencia lejana de la intervención ${ }^{57}$. Los Tribunales Regionales Superiores ${ }^{58}$ con frecuencia también exigen una información "drástica" o "desconsiderada" 59.

Solo cuando se cumplan los requisitos relativos a la información ${ }^{60}$, resulta válido el consentimiento dado por el paciente así extensamente informado. En lugar del término convencional de informed consent, en

${ }^{52}$ En este sentido con relación al Derecho chileno Pizarro (2008), p. 176 (“existe un deber mayor de información al paciente"; "no sólo los riesgos normales sino, también, aquéllos excepcionales”); en cuanto al Derecho español véase Pérez (2010), p. 157, “en el ámbito de la medicina satisfactiva el nivel de información que hay que suministrar es mucho mayor" y pp. 161-163.

${ }^{53}$ LAUfS, KatzenMeIER y LipP (2009), capítulo v, cons. 20², véase, por ejemplo, BGH (2006b), p. 2108; BGH (1990), p. 2349 "requisitos muy estrictos".

${ }^{54}$ Opinión contraria Lorz (2007), p. 175, que se basa en un concepto de indicación - a mi juicio demasiado amplio-, otra opinión crítica DAMM (2010), p. 653, según la cual debemos seguir centrándonos en la misión curativa tradicional como patrón paradigmático.

${ }^{55}$ Esto posiblemente se manifiesta también en BGH (1980), p. 633: En tal caso [falta de cualquier indicación urgente para la operación con alto riesgo de fracaso] es especialmente importante dejar la decisión sobre la operación al criterio del paciente.

${ }^{56}$ Otra opinión diferente Lorz (2007), pp. 101 y 252, que defiende la armonización del régimen de legitimación de la medicina del deseo y la medicina curativa y postula el principio de la información orientada al paciente.

${ }^{57}$ En este sentido BGH (2006b), p. 2108.

${ }^{58}$ En este sentido también los materiales legales relativos a la Ley de Derechos del Paciente -aunque en esta, de forma sistemática, referenciada incorrectamente-, véase Boletín oficial del Bundestag (2012), p. 25.

${ }^{59}$ Véase OLG Düsseldorf (2002), p. 386; OLG Düsseldorf (1997), p. 61 “especialmente desconsiderada"; OLG Hamm (2006), p. 1512 "drástica y desconsiderada”; conforme DAmm (2010), p. 652, aunque critica el uso del término 'desconsiderada' (p. 651).

${ }^{60}$ Detalles al respecto más adelante III. 5 a). 
el presente contexto se estima más adecuada la denominación intensely informed consent.

\section{Estándar reconocido de la medicina especializada en la cirugía estética plástica}

En el ámbito de la cirugía estética debe cumplirse el estándar vigente en el ramo especial de la cirugía estética plástica ${ }^{61}$. En 1992 se incluyó en el MWBO del BÄK el título de médico especialista Facharzt für Plastische Chirurgie (médico especialista de cirugía plástica), modificado desde 2005 en Facharzt für Plastische und Ästhetische Chirurgie (médico especialista de cirugía plástica y estética ${ }^{62}$. La formación especializada de los médicos aprobados antes de esta fecha comprende en este ramo especial un periodo de seis $^{63}$ años $^{64}$.

Si un médico, llevando a cabo una intervención de cirugía estética, queda por debajo del mencionado estándar reconocido de la medicina especializada, incurre por regla general con un error de tratamiento. Entre las categorías de errores más frecuentes del ámbito en cuestión cuentan la elección del método incorrecto ${ }^{65}$, errores al realizar la intervención quirúrgica $^{66}$ e insuficiencia de la llamada información terapéutica ${ }^{67}$.

No obstante, el error de tratamiento puede radicar en el mero hecho de que se realice la intervención, si la misma en el caso concreto es contraindicada. En este contexto es de especial importancia el problema de la dismorfofobía corporal (también denominada trastorno dismórfico corporal) que sobre todo en la cirugía estética, se presenta no con poca frecuencia ${ }^{68}$. Por regla general, esta enfermedad constituye un criterio de exclusión de una intervención meramente estética, ya que los pacientes

${ }^{61}$ Véase, por ejemplo, OLG Stuttgart (2002), p. 463.

${ }^{62}$ Ahora sección B, 7.6 MWBO.

${ }^{63}$ De ellos dos años de formación básica de cirugía.

${ }^{64}$ Aparte de ello, los especialistas de otorrinolaringología y de cirugía oral y maxilofacial pueden adquirir, adicionalmente a su competencia como médico especialista y después de pasar una formación especializada con una duración de dos años, la denominación complementaria de Plastische Operationen (operaciones de cirurgía plástica) para intervenciones en la zona de la cabeza y del cuello.

${ }^{65}$ Por ejemplo, colocación de un implante mamario en vez del estiramiento de la capa cutánea para corregir un hundimiento pectoral.

${ }^{66}$ Por ejemplo, sección de vasos sanguíneos que abastecen los pezones de sangre.

${ }^{67} \mathrm{El}$ médico que omite mencionar, por ejemplo, que la paciente no debe hacer actividades deportivas durante un tiempo determinado después de la intervención con el fin de garantizar el resultado del tratamiento.

${ }^{68}$ Según Lorz (2014), p. 112 con más pruebas: 7 \% de los que se someten a operaciones de cirugía estética. 
afectados presentan una obsesión de repetición y una medida estética podría, incluso, agravar aún más el trastorno ${ }^{69}$. Lo indicado en tales constelaciones son psicoterapias, tratamientos con medicamentos para curar la dismorfofobía corporal o ambos.

\section{Decisiones seleccionadas de la jurisprudencia}

En Alemania el BGH y, en particular los Tribunales Regionales Superiores han concretizado con más detalles las obligaciones de médicos y clínicas en materia de la cirugía estética sin indicación médica. A continuación se esbozarán algunos aspectos por medio de varias sentencias.

\section{a) Información}

Antes de practicar intervenciones estéticas se exige una información extensa o "desconsiderada" de los pacientes ${ }^{70}$, y más aun cuando se trata de intervenciones complejas que implican un considerable riesgo de que no se logre el resultado intentado. Incluso, en caso de una operación LASER experimental, que por su finalidad es comparable con una operación meramente cosmética (objetivo: sustitución de lentes), hay que decirle "sin rodeos [a la paciente], que si tiene mala suerte podría perder la vista por completo" ${ }^{\text {. }}$

Por cierto, en la literatura se afirma que, según la jurisprudencia, la información "desconsiderada" sobre riesgos en su caso debe incluir también la presentación de fotografías, por ejemplo, de deformaciones resultado de intervenciones fracasadas ${ }^{72}$. De hecho y contrario a esto, en la jurisprudencia publicada se encuentran -según se ha podido apreciar- muy aislados ejemplos de decisiones de los Tribunales Regionales Superiores, algunas de ellas dictadas hace más de treinta años, en las que realmente se exigió la utilización de imágenes en color (en su caso obtenidas de la literatura especializada) para ilustrar el estado corporal final o posibles queloídes $^{73}$. Entretanto, el Tribunal Regional Superior de Múnich rechaza en forma expresa tal obligación porque -según argumenta- la información no debe tener el objetivo de espantar a los pacientes, sino que debe servir para facilitar una decisión en responsabilidad propia ${ }^{74}$.

La información antes de una liposucción por razones meramente estéticas debe incluir, entre otros, los excesos de piel y la formación de

\footnotetext{
${ }^{69}$ Véase ejemplos de la jurisprudencia más adelante III. 5. b).

${ }^{70}$ Pruebas más atrás en la n. $\mathrm{N}^{\circ} 59$.

${ }^{71}$ Así expresamente OLG Köln (2009b), p. 718.

${ }^{72}$ Véase LoRz (2007), pp. 151-152.

${ }^{73}$ Véase OLG Hamburg (1982), p. 581; OLG München (1985), pp. 188-189.

${ }^{74}$ Véase OLG München (2010), p. 415.
} 
hoyos en la misma como posibles riesgos de información obligada ${ }^{75}$. Como las perspectivas de éxito y el riesgo de fracaso son de importancia fundamental para la decisión sobre la operación, la paciente puede esperar una indicación correcta al menos en cuanto a la magnitud del riesgo de defectos estéticos ${ }^{76}$. En el caso de una abdominoplastía se debe informar acerca del largo de la cicatriz, sensaciones de tensión y trastornos de la sensibilidad a largo plazo, entre otros; si esta información no es dada a tiempo -en el asunto litigioso, en la noche anterior al día en que ocurre la intervención- la misma se considerará tardía y, por tanto, el consentimiento será inválido ${ }^{77}$.

Antes de una intervención cosmética, para aumentar los senos, la paciente debe ser advertida con claridad sobre el riesgo de perjuicios por dolores durante toda la vida a causa de sobreextensiones de los músculos pectorales $^{78}$. Y, aunque la indicación de una reducción de senos sea solo en parte cosmética, se debe informar también sobre la posibilidad de que debido a tejido graso remanente debajo del sobaco puede resultar necesaria una intervención correctiva ${ }^{79}$. Además, la parte tratante debe informar también desconsideradamente sobre la imposibilidad de alcanzar el resultado deseado por la paciente (evitar cicatrices manteniendo igual el tamaño de los senos ${ }^{80}$.

b) Errores de tratamiento

Como error de tratamiento fue calificado, por ejemplo, que en una corrección de pechos la incisión se encontraba a una distancia de aproximadamente $3 \mathrm{~cm}$ del pliegue del seno ${ }^{81}$. Al contrario, no fue considerado un error de tratamiento por parte del tribunal una leve diferencia entre la altura de ambos pechos después de colocar implantes mamarios por estar todavía dentro del margen de dispersión de procesos curativos biológicos normales ${ }^{82}$.

Antes de realizar intervenciones meramente cosméticas es importante comprobar si existen eventuales contraindicaciones, en particular en cuanto a una actitud psíquica neurótica de un paciente (dismorfofobía corporal). Un indicio de este trastorno puede ser que este se ha some-

${ }^{75}$ Véase OLG Köln (2009a), p. 1607.

${ }^{76} \mathrm{Ibid}$., indicación a la petición de cuantificación de la paciente según la experiencia propia del médico y sus colegas.

${ }^{77}$ En este sentido OLG Frankfurt/Main (2005), p. 296.

${ }^{78}$ Véase OLG Hamm (2006), p. 1512.

${ }^{79}$ Véase OLG Stuttgart (1999), pp. 904-905, así como en caso de liposucción OLG Düsseldorf (2003), pp. 1579-1580.

${ }^{80}$ LG München I (2013), p. 1315 (no firme).

${ }^{81}$ OLG Düsseldorf (1988), p. 1297.

${ }^{82}$ En detalle, véase OLG Sachsen-Anhalt (2012), citado según Juris, cons. $6^{\circ}$ y $8^{\circ}$. 
tido ya a múltiples operaciones previas, sin que se considere indicio de trastorno que se hayan realizado entre dos y tres intervenciones estéticas previas $^{83}$, ni tampoco cuando se desee una corrección que aplicando un criterio objetivo no se estima indicada ${ }^{84}$. Sin embargo, puede ser aconsejable consultar al médico que le atendió previamente ${ }^{85}$. Aparte de esto, puede ser indicio de una dismorfofobía corporal también si el paciente presenta ansias extraordinarias. Por ejemplo, como claro indicio en este sentido ha sido calificado el deseo para corregir una asimetría insignificante de los labios vulvares ${ }^{86}$. Por último, las depresiones se consideran una contraindicación relativa para realizar una intervención deseada de carácter meramente estética ${ }^{87}$.

\section{Reflexiones complementarias acerca de aspectos de remuneración}

La remuneración de los servicios profesionales de los médicos se rige, por principio, por el GOÄ ${ }^{88}$. Según la doctrina sentada por el BGH, estos están sujetos a las disposiciones del GOÄ, incluso cuando se trata de la liquidación privada de operaciones cosméticas sin indicación médica ${ }^{89}$. Por cierto, en el $\S 2$ del GOÄ se permite concertar para casos particulares acuerdos individuales escritos relativos al importe del tipo de aumento ${ }^{90}$, pero las condiciones establecidas son muy restrictivas y los márgenes de tolerancia son limitados. Además, en las operaciones de cirugía estética no tiene lugar el beneficio de exención del impuesto sobre la venta que sí es aplicable a los tratamientos curativos ${ }^{91}$.

Finalmente, en el ámbito de la cirugía estética puede adquirir más importancia la información económica de los pacientes. Estos deben ser informados por el médico de forma inequívoca de que el seguro de enfermedad posiblemente no va a asumir los gastos de la intervención ${ }^{92}$, siempre y cuando esto no sea del conocimiento del paciente ${ }^{93}$. Aparte de ello, la remuneración se debe por principio, aun en los casos en que no

${ }^{83}$ OLG Düsseldorf (2000), p. 1381.

${ }^{84}$ OLG Köln (1999), p. 1372.

${ }^{85}$ OLG Düsseldorf (2000), p. 1381.

${ }^{86}$ Véase OLG Düsseldorf (1984), p. 684.

${ }^{87}$ Véase OLG Hamm (2009), citado según Juris cons. $39^{\circ}$ a $42^{\circ}$ (liposucción).

${ }^{88}$ Véase $\S 1$ párrafo 1 GOÄ.

${ }^{89}$ Véase BGH (2006a), pp. 1880-1881.

${ }^{90}$ Pero no un precio global, véase también BGH (2006a), 1881; OLG Stuttgart (2002), p. 463.

${ }^{91}$ Detalles al respecto BFH (2014).

${ }^{92}$ En detalle, véase Martis y Winkhart (2014), cons. A $824^{\circ}$ a $825^{\circ}$.

${ }^{93}$ Véase OLG Stuttgart (2002), p. 463. 
se logre el resultado intentado de una intervención meramente cosmética realizada según estándar ${ }^{94}$.

\section{Conclusiones}

El contrato civil de prestaciones de la cirugía estética sin indicación médica es clasificado como contrato de servicio, salvo que -en un caso excepcional e improbable- el médico prometa clara e indudablemente un resultado. Por consiguiente, en el Derecho alemán los médicos no les deben a los pacientes un resultado, sino solamente su esfuerzo esmerado por conseguir dicho resultado.

No obstante, a cargo de la parte tratante ha de considerarse que toda intervención médica -sea con fines curativos o por motivos estéticos- es calificada como lesión corporal. Por eso en el juicio civil corresponde a los médicos la carga de alegación y prueba de la debida información del paciente y del consentimiento válido del mismo como hecho justificativo de la lesión corporal (informed consent). Esta carga pesa tanto más en el ámbito de la cirugía estética en cuanto los juzgados establecen requisitos muy rigurosos con respecto a la información del paciente. Mediante este endurecimiento se intenta revaluar la declaración de su consentimiento en forma de un aumento de la información del mismo (aquí denominado intensely informed consent) con el fin de compensar en la medida de lo posible la falta de indicación médica de una intervención meramente estética. La autonomía del paciente (volenti non fit iniuria), que cada vez va teniendo más importancia, es acompañada por un amplio fundamento de información que debe ser creado por el médico antes de la intervención.

Por otro lado, la autonomía del paciente se ve limitada particularmente cuando existe una contraindicación. Este puede ser el caso cuando el deseo de "embellecimiento" se deriva de una actitud psíquica neurótica del paciente (dismorfofobía corporal). Dado el caso, el médico no debe llevar a cabo la intervención deseada -para proteger tal paciente limitado en su capacidad de autodeterminación, así como para evitar que él mismo incurra en responsabilidad frente a esta.

\section{Bibliografía CITADA}

Barcia Lehmann, Rodrigo (2010). "Algunas consideraciones de la relación de causalidad material y jurídica en la responsabilidad civil médica, en Cuadernos

${ }^{94}$ Véase detalles del destino de la obligación de remuneración MARTiS y WinKHART (2014), cons. A $430^{\circ}$ a $433^{\circ}$. 
de Análisis Jurídicos, Responsabilidad Médica. Santiago: Ediciones Universidad Diego Portales. Colección Derecho Privado, vol. VI.

Cárdenas Villarreal, Hugo A. (2010). "La cobertura dogmática de la recepción jurisprudencial de la distinción obligaciones de medios/obligaciones de resultado (una aproximación a través de casos de responsabilidad médica", en Cuadernos de Análisis Jurídicos, Responsabilidad Médica. Santiago: Ediciones Universidad Diego Portales. Colección Derecho Privado, vol. vI.

DAmm, Reinhard (2010). "Ästhetische Chirurgie und Medizinrecht - Normstrukturen, Regelungsprobleme und Steuerungsebenen”, in Gesundheitsrecht (GesR). Volume 9, No 12, Kölhn.

De La Maza Gazmuri, Iñigo (2010). "Consentimiento informado y relación de causalidad”, en Cuadernos de Análisis Jurídicos, Responsabilidad Médica. Santiago: Ediciones Universidad Diego Portales. Colección Derecho Privado, vol. vi.

Domínguez Hidalgo, Carmen (2010). "El problema de la culpa presunta contractual y las obligaciones de medio y obligaciones de resultado: sus implicancias para la responsabilidad médica", en Cuadernos de Análisis Jurídicos, Responsabilidad Médica. Santiago: Ediciones Universidad Diego Portales. Colección Derecho Privado, vol. vI.

EвERbACH, Wolfram H. (2008), "Die Verbesserung des Menschen - Tatsächliche und rechtliche Aspekte der wunscherfüllenden Medizin", in Medizinrecht, Vol. 26. $\mathrm{N}^{\mathrm{O}}$ 6. Berlin-Heidelberg.

Figueroa García Huidobro, Rodolfo (2010). "Autonomía de los pacientes y responsabilidad de los médicos", en Cuadernos de Análisis Jurídicos, Responsabilidad Médica. Santiago: Ediciones Universidad Diego Portales. Colección Derecho Privado, vol. VI.

FrÖDING, Veronique (2015). "PIP Brustimplantate: Warum das Bernfungsgericht Aix en Provence die Haftung des tüv abgelehuthat", in Medizinprodukterecht. Vol. 15. $\mathrm{N}^{\circ}$ 5. Baden-Baden.

Geiß, Karlmann und Hans-Peter Greiner (2014). Arzthaftpflichtrecht. Vol. 7. München.

Katzenmeier, Christian (2014). "Patientenrechte und Arzthaftung", in Egon LoRENZ (ed.), Karlsruher Forum 2013: Patientenrechte und Arzthaftung. Karlsruhe: Verlag Versicherungswirtschaft.

Katzenmeier, Christian (2002). Arzthaftung. Tübingen: Mohr Siebeck.

Laufs, Adolf, Christian Katzenmeier und Volker Lipp (2009). Arztrecht. Vol. 6. München.

Laufs, Adolf und Bernd-Rüdiger Kern (2010). Handbuch des Arztrechts. Vol. 4. München.

LoRz, Sigrid (2007). Arzthaftung bei Schönheitsoperationen. Berlin: Duncker \& Humblot.

Martis, Rüdiger und Martina Winkhart-Martis (2014). Arzthaftungsrecht. Fallgruppenkommentar. Vol. 4. Köln. 
Pérez García, Máximo Juan (2010). "La responsabilidad civil médico-sanitaria en el Derecho español: significado y alcance del consentimiento informado y el deber de información, en Cuadernos de Análisis Jurídicos, Responsabilidad Médica. Santiago: Ediciones Universidad Diego Portales. Colección Derecho Privado, vol. VI.

Pizarro Wilson, Carlos (2013). "La obligación de resultado en el contrato médico. Hacia una responsabilidad objetiva", en Estudios de Derecho Civil IX, Valdivia: Universidad Austral.

PiZarro Wilson, Carlos (2011). “Obligaciones y responsabilidad civil”, en Revista Chilena de Derecho Privado, $\mathrm{N}^{\circ} 17$, Santiago.

Pizarro Wilson, Carlos (2010). "Controversias jurisprudenciales de la responsabilidad de los servicios públicos de salud”, en Cuadernos de Análisis Jurídicos, Responsabilidad Médica. Santiago: Ediciones Universidad Diego Portales. Colección Derecho Privado, vol. vI.

Pizarro Wilson, Carlos (2008). "Responsabilidad profesional médica: Diagnóstica y perspectivas", en Cuadernos de Análisis Jurídicos. Regímenes especiales de responsabilidad civil. Santiago: Ediciones Universidad Diego Portales, Colección Derecho Privado, vol. IV.

SpickHoff, Andreas (ed.) (2014). Medizinrecht-Kommentar, Vol. 2. München.

Wiegner, Matthias (2010). "Materielle und prozessuale Voraussetzungen der Arzthaftung in Spanien”, in Cross Boarder Treatment-Die Arzthaftung wird europäisch, MedR Schriftenreihe Medizinrecht. Berlin-Heidelberg.

\section{Otros documentos utilizados}

Boletín oficial del bundestag [Bundestags-Drucksache] (2012). $\mathrm{N}^{\circ} 17 / 10488$ de 15 agosto de 2012. Disponible en http://dip21.bundestag.de/dip21/btd/17/104/1710488. pdf. [fecha de consulta 7 de abril de 2015].

\section{Normas citadas}

$B G B$, escriturado: 18 de agosto de 1896, anunciado: 2 de enero de 2002 (Bundesgesetzblatt I, p. 42, 2909; 2003 I, p. 738), último cambio: Art. 1 ley de 22 de julio de 2014 (Bundesgesetzblatt I, p. 1218).

$S G B V$ - Gesetzliche Krankenversicherung, art. 1 ley de 20 de diciembre 1988 (Bundesgesetzblatt I pp. 2477, 2482), último cambio: Art. 2a ley de 17 de diciembre de 2014 (Bundesgesetzblatt I, p. 2222).

GOÄ, último cambio: Art. 17 Ley de 4 de diciembre de 2001 (Bundesgesetzblatt I, p. 3320)

Gesetz zur Verbesserung der Rechte von Patientinnen und Patienten („Patientenrechtegesetz“) de 20 de febrero de 2013 (Bundesgesetzblatt I 2013, p. 277).

MWBO de Consejo General de Colegios Médicos (BÄK), 28 de junio de 2013. 


\section{Jurisprudencia citada}

BFH (2014), 4 de diciembre de 2014, N v R 16/12, in Deutsches Steuerecht (DStR) Vol. 53. $\mathrm{N}^{\mathrm{o}}$ 8. München.

BGH (2015), 9 de abril de 2015, N VII ZR 36/14, (no publicado).

BGH (2012), 19 de junio de 2012, N $^{\circ}$ vi ZR 77/11, in NJW, Vol. 65. No 36. München.

BGH (2011), 25 de octubre de 2011, $\mathrm{N}^{\circ}$ VI ZR 139/10, in NJW, Vol. 65. $\mathrm{N}^{\circ} 4$. München. 2012.

BGH (2006a), 23 de marzo de 2006, $\mathrm{N}^{\circ}$ III ZR 223/05, in NJW, Vol. 59. $\mathrm{N}^{\circ} 26$. München. 2006.

BGH (2006b), 14 de marzo de 2006, N $^{\circ}$ vi ZR 279/04, in NJW, Vol. 59. No 29. München. 2006.

BGH (1995), 21 de noviembre de 1995, $\mathrm{N}^{\circ}$ vi ZR 341/94, in NJW, Vol. 49. $\mathrm{N}^{\circ} 12$. München. 1996.

BGH (1990), 6 de noviembre de 1990, $\mathrm{N}^{\circ}$ vi ZR 8/90, in NJW, Vol. 44. $\mathrm{N}^{\circ} 37$. München. 1991.

BGH (1980), 23 de septiembre de 1980, $\mathrm{N}^{\circ}$ VI ZR 189/79, in NJW, Vol. 34. $\mathrm{N}^{\circ} 12$. München. 1981.

BGH (1979), 23 de octubre de 1979, N $^{\circ}$ VI ZR 197/78, in NJW, Vol. 33. N $^{\circ} 12$. München. 1980.

BGH (1958), 9 de diciembre de 1958, N $^{\circ}$ vi ZR 203/57, in Juris.

Cour D’Applel d'aix en Provence (2015), 2 de julio de 2015. No 2015/335 (no publicado).

LG. München I (2013), 31 de julio de 2013, Nº 9 O 25313/11 (no firme), in Versicherungsrecht (VersR). Vol. 64. $\mathrm{N}^{\circ}$ 30. Karlsruhe.

OLG Düsseldorf (2003), 20 de marzo de 2003, $\mathrm{N}^{\circ} 8$ U 18/02, in Versicherungsrecht (VersR). Vol. 54. No 36. Karlsruhe.

OLG Düsseldorf (2002), 21 de marzo de 2002, N 8 U 117/01, in Versicherungsrecht (VersR). Vol. 55. N 9, Karlsruhe. 2004.

OLG Düsseldorf (2000), 19 de octubre de 2000, $\mathrm{N}^{\circ} 8 \mathrm{U}$ 116/99, in Versicherungsrecht (VersR). Vol. 52. No 32, Karlsruhe. 2001.

OLG Düsseldorf (1997), 13 de octubre de 1997, Nº 8 U 102/96, in Versicherungsrecht (VersR). Vol. 50, № 2, Karlsruhe. 1999.

OLG Düsseldorf (1988), 5 de mayo de 1988, Nº 8 U 130/86, in Versicherungsrecht (VersR). Vol. 39. N $^{\circ}$ 48, Karlsruhe.

OLG Düsseldorf (1984), 15 de noviembre de 1984, $\mathrm{N}^{\circ} 8 \mathrm{U}$ 189/83, in NJW, Vol. 38. N 12. München. 1985.

OLG Frankfurt/Main (2005), 11 de octubre de 2005, $\mathrm{N}^{\circ} 8 \mathrm{U}$ 47/04, in Medizinrecht (MedR). Vol. 24. No 5. Berlin-Heidelberg. 2006. 
OLG Hamburg (2005), 29 de diciembre de 2005, $\mathrm{N}^{\circ} 1 \mathrm{~W}$ 85/05, in Monatsschrift für deutsches Recht (MDR). Vol. 59. No 15. Köln. 2006.

OLG Hamburg (1982), 5 de marzo de 1982, $\mathrm{N}^{\circ} 1$ U 5/81, in Monatsschrift fïr deutsches Recht (MDR). Vol. 36. No 7. Köln.

OLG Hamm (2009), 25 de febrero de 2009, $\mathrm{N}^{\circ} 3$ U 51/08, in Juris.

OLG Hamm (2006), 29 de marzo de 2006, N 3 U 263/05, in Versicherungsrecht (VersR). Vol. 57. No $^{\mathrm{o}}$ 32. Karlsruhe.

OLG Köln (2009a), 21 de diciembre de 2009, № 5 U 52/09, in Versicherungsrecht (VersR). Vol. 61. No 35. Karlsruhe. 2010

OLG Köln (2009b), 12 de agosto de 2009, N 5 U 47/09, in Medizinrecht (MedR). Vol. 28. $\mathrm{N}^{\mathrm{O}}$ 10. Berlin-Heidelberg. 2010

OLG Köln (1999), 3 de febrero de 1999, № 5 U 118/98, in Versicherungsrecht (VersR). Vol. 50. $\mathrm{N}^{\mathrm{O}}$ 32. Karlsruhe.

OLG München (2010), 22 de abril de 2010, N 1 U 3807/09, in Gesundheitsrecht (GesR). Vol. 9. No 8. Köln.

OLG München (1985), 19 de septiembre de 1985, № 24 U 117/85, in Medizinrecht (MedR). Vol. 6. No 4. Berlin-Heidelberg. 1988.

OLG Sachsen-Anhalt (2012), 16 de febrero de 2012, $N^{\circ} 1$ U 88/11, in Juris.

OLG Stuttgart (2002), 9 de abril de 2002, N 14 U 90/01, in Versicherungsrecht (VersR). Vol. 54. No 11. Karlsruhe. 2003.

OLG Stuttgart (1999), 20 de julio de 1999, N 14 U 1/99, in Neue Juristische Wochenschrift-Rechtsprechungsreport(NJW-RR). Vol. 15. No 13. München. 2000.

OLG Zweibrücken (2014), 30 de enero de 2014, $\mathrm{N}^{\circ} 4$ U 66/13, in Medizinprodukterecht (MPR). Vol. 14. No 2. Baden-Baden.

RG (1894), 31 de mayo de 1894, $\mathrm{N}^{\circ} 1406 / 94$, in RGSt 25. Berlin.

RG (1908), 27 de mayo de 1908, $\mathrm{N}^{\circ}$ VI 484/07, in RGZ 68, Berlin.

SG Berlin (2013), 10 de diciembre de 2013, N S 182 KR 1747/12, in Juris.

Tribunal de Commerce Toulon (2013), 14 de noviembre de 2013, Nº 2011F00517, in Medizinprodukterecht MPR. Vol. 14. No 1. Beden-Beden. 2014. 